\section{Sensibilidade do teste parasitológico em imprints de baço, medula óssea e linfonodo para o diagnóstico da leishmaniose de acordo com o número de avaliadores}

\author{
Sensitivity of parasitological test in spleen, bone marrow and \\ lymph node imprints for leishmaniasis diagnosis according to \\ the number of evaluators
}

\author{
Marcus Vinícius Caetano de Sousa', Matias Pablo Juan Szabó2 , Geórgia Mode Magalhães³, \\ Rodrigo Pereira de Queiroz ${ }^{2}$, Igor Paula de Castro', Endrigo Gabellini Leonel Alves ${ }^{4}$, \\ Alessandra Aparecida Medeiros ${ }^{2} \&$ Tais Meziara Wilson* \\ 'Médicos veterinários, Mestrandos, Bolsistas CAPES. Programa de Pós-graduação em Ciências Veterinárias, Faculdade de \\ Medicina Veterinária, Universidade Federal de Uberlândia - UFU, Uberlândia, MG, Brasil \\ 2Médicos veterinários, DSc. Laboratório de Patologia Animal, Faculdade de Medicina Veterinária, Universidade Federal de \\ Uberlândia - UFU, Uberlândia, MG, Brasil \\ ${ }^{3}$ Médica veterinária, DSc. Universidade de Franca - Unifran, Franca, SP, Brasil \\ ${ }^{4}$ Médico veterinário, DSc. Curso de Medicina Veterinária, Universidade de Uberaba - UNIUBE, Uberaba, MG, Brasil
}

\section{Resumo}

A Leishmaniose Visceral é considerada pela Organização Mundial de Saúde uma das seis endemias prioritárias no mundo, sendo que os cães atuam como reservatórios naturais do parasito. O diagnóstico da enfermidade nos cães é discutido e o propósito do presente trabalho foi de verificar o método parasitológico como meio de diagnóstico da Leishmaniose Visceral Canina utilizando amostras de baço, medula óssea e linfonodo, além de correlacionar com a sintomatologia clínica dos animais e analisar a sensibilidade do diagnóstico parasitológico realizado por um e dois observadores. Foram utilizados 59 cães obtidos pelo Centro de Controle de Zoonoses de Uberlândia - MG. Os animais foram examinados clinicamente antes da eutanásia e classificados em assintomáticos (grupo 1) ( $n=6$ ), oligossintomáticos (grupo 2) (n=34) e sintomáticos (grupo 3) ( $n=19$ ). Trinta e três (55,93\%) animais foram positivos e destes 20 (60,60\%) pertenciam ao grupo 2,11 (33,33\%) ao grupo 3 e 2 (6,06\%) ao grupo 1. Em relação aos locais de coleta analisados obteve-se 73 (41,24\%) amostras positivas, sendo 28 (38,35\%) de medula óssea, 25 (34,24\%) de baço e 20 (27,39\%) de linfonodo. Quando a leitura foi realizada por um observador obteve-se $13(22,03 \%)$ animais positivos, e com dois observadores este número foi de $33(55,93 \%)$, sendo este aumento na sensibilidade significativo estatisticamente. Não houve diferença estatística entre o número de animais distribuídos entre os grupos com sintomatologia clínica e tampouco quanto ao local puncionado. Recomenda-se a leitura realizada por dois observadores para aumento na sensibilidade do teste parasitológico.

Palavras-chave: sensibilidade, medula óssea, baço, linfonodo, dois observadores.

\begin{abstract}
Visceral Leishmaniasis is considered by the World Health Organization as one of the six priority endemic diseases in the world, once that dogs are natural reservoirs of the parasite. The diagnosis of this disease in dogs is discussed and the purpose of this work was to study the method of parasitological diagnosis of canine visceral leishmaniasis using samples of spleen, bone marrow and lymph node, and to correlate with the clinical signs of the animals and examine the sensitivity parasitological diagnosis performed by one and two observers. There were obtained 59 dogs, by Uberlandia's Zoonosis Center Control in Minas Gerais State. The animals were clinically examined before the euthanasia and classified as asymptomatic (group 1) (n=6), mildly symptomatic (group 2) (n=34) and symptomatic (group 3) (n=19).Thirty-three (55.93\%) were positive, 20 of those (60.60\%) were belonged to group 2,11 of those (33.33\%) were to group 3 and 2 (6.06\%) were in the group 1.In relation to the organ collected was obtained that 73 (41.24\%) were positive samples, 28 (38.35\%) from bone marrow, 25 (34.24\%) from spleen and 20 (27.39\%) from lymph node. When the microscope exam were done by one of the observer, he obtained 13 (3.22\%) of positive animals, however the same method evaluated by the second observer this number increased to 33 (55.93\%), and this increase was statistically significant. There was no statistical difference between the number
\end{abstract}

Como citar: Sousa, M. V. C., Szabó, M. P. J., Magalhães, G. M., Queiroz, R. P., Castro, I. P., Alves, E. G. L., Medeiros, A. A., \& Wilson, T. M. (2017). Sensibilidade do teste parasitológico em imprints de baço, medula óssea e linfonodo para o diagnóstico da leishmaniose de acordo com o número de avaliadores. Brazilian Journal of Veterinary Medicine, 39(3), 176-181. doi: 10.29374/2527-2179.bjvm027417

Fonte de financiamento: Fundação de Amparo à Pesquisa do Estado de Minas Gerais (FAPEMIG) (Projeto APQ-01905-10) Financiamento de bolsas de mestrado pela CAPES.

Conflito de interesses: Os autores declaram não haver conflito de interesses que precisam ser informados.

Recebido: Março 11, 2017.

Aceito: Setembro 30, 2017

O estudo foi realizado na Universidade Federal de Uberlândia - UFU, Uberlândia, MG, Brasil.

\section{*Correspondência}

Tais Meziara Wilson

Programa de Pós-graduação em Ciências Veterinárias, Faculdade de Medicina Veterinária, Universidade Federal de Uberlândia - UFU

Av. Ceará, s/n, Bloco 2T, Campus Umuarama CEP 38400-902 - Uberlândia (MG), Brasil E-mail: taismeziara@gmail.com

Copyright Sousa et al. Este é um artigo publicado em acesso aberto (Open Access) sob a licença Creative Commons Attribution Non-Commercial, que permite uso, distribuição e reprodução em qualquer meio, sem restrições desde que sem fins comerciais e que o trabalho original seja corretamente citado. 
of animals distributed among the groups with clinical symptoms, neither the place where the imprints where done. Considering the increase of the sensitivity by the parasitological test, it is recommended that the analyses' results should be to be obtained by two observers.

Keywords: sensitivity, bone marrow, spleen, lymph node, two observers.

\section{Introdução}

A Leishmaniose Visceral (LV) é uma zoonose causada pelo protozoário Leishmania infantum, sendo considerada pela Organização Mundial de Saúde (World Health Organization, 2010) uma das seis endemias prioritárias no mundo. O método de transmissão é pelo vetor do gênero Lutzomyia, e os cães atuam como reservatórios naturais do parasito (Silva et al., 2009).

De acordo com a literatura, nenhum teste diagnóstico para Leishmaniose Visceral Canina (LVC) tem 100\% de sensibilidade e especificidade (Gontijo \& Melo, 2004; Silva, 2007; Brasil, 2014). As técnicas sorológicas recomendadas pelo Ministério da Saúde para o inquérito canino são a imunofluorescência indireta (RIFI) e ELISA (Brasil, 2014), porém estas podem apresentar resultado falso-positivo por reação cruzada o que acarreta a eutanásia de cães que não estão infectados. Assim, a escolha de um teste de diagnóstico preciso e reprodutível, capaz de suportar estratégias de controlo e vigilância em caninos, é fundamental (Peixoto et al., 2015).

A pesquisa direta de parasitas em tecidos tem sido considerada como diagnóstico definitivo para leishmaniose (Solano-Gallego et al., 2011; Abreu et al., 2015) sendo a análise de esfregaços de material colhido por meio de punção de baço, medula óssea e linfonodos um método eletivo para se identificar a forma amastigota em microscopia óptica (Troncarelli et al., 2009). Saridomichelakis et al. (2005) observaram o linfonodo como o melhor local para visualização do parasita, já Andrade et al. (2006) observaram que além do linfonodo o baço também é um bom local para se realizar o imprint, havendo portanto divergência entre autores quanto ao melhor local a ser puncionado para se obter um diagnóstico mais preciso.

O teste parasitológico possui 100\% de especificidade e pode auxiliar no diagnóstico da LVC eliminando resultados falso-positivos e, como consequência, a eutanásia de animais livres da doença. Por outro lado, os animais assintomáticos são considerados fonte de infecção sendo um problema para a saúde pública. É uma técnica rápida, com baixo custo econômico e de fácil execução, porém é necessário ter conhecimento do melhor local para a coleta de material e bom treinamento do observador.

Diante das dificuldades no diagnóstico desta zoonose em cães, objetiva-se neste trabalho verificar qual o local mais adequado para coleta de material no exame parasitológico, avaliar a sensibilidade do teste de acordo com o número de observadores, além de associar este resultado com a sintomatologia clínica.

\section{Materiais e métodos}

Foram utilizados 59 cães sororreagentes para os testes de Imunofluorescência Indireta (RIFI) (ponto de corte $\geq 1: 40$ ) e ELISA de acordo com o preconizado pelo Ministério da Saúde, provenientes do Centro de Controle de Zoonose (CCZ) do município de Uberlândia onde os exames foram realizados. No CCZ os animais foram submetidos ao exame clínico padrão seguindo a ficha clínica do Hospital Veterinário da Universidade Federal de Uberlândia (HV-UFU). De acordo com Mancianti et al. (1988) os cães foram classificados em 3 grupos, sendo grupo 1 composto por animais assintomáticos (sem sinais clínicos), grupo 2 de oligossintomáticos (um a três sinais clínicos) e grupo 3 de sintomáticos (> 3 sinais clínicos). Os principais sinais clínicos observados para a divisão dos grupos foram: onicogrifose, áreas de alopecia, linfoadenomegalia, hepatoesplenomegalia e uveíte.

Após o exame clínico, os animais foram eutanasiados e encaminhados ao Laboratório de Patologia Animal do HV-UFU onde se procedeu à necropsia dos mesmos. Foram confeccionados imprints em lâminas de microscopia por duplicata de baço, medula óssea (Secção de Fêmur) e linfonodos poplíteos sendo posteriormente coradas pelo método Panótico Rápido (Renylab ${ }^{\circledR}$. Os animais compreendiam cães de várias idades, sexo e raças, sendo a maioria sem raça definida.

O exame parasitológico foi realizado através da pesquisa de formas amastigotas, em microscopia óptica, utilizando-se óleo de imersão, objetiva de 100 vezes, observando-se 100 campos. As amostras 
foram submetidas à leitura por dois observadores com experiência em identificar as formas amastigotas e os critérios estabelecidos para considerar uma amostra positiva foi visualização das três estruturas básicas da forma amastigota do parasita (núcleo, citoplasma e cinetoplasto), estruturas íntegras, independente da carga parasitária e se estavam livres ou dentro de macrófagos .

O cálculo da sensibilidade do teste utilizando-se um ou dois observadores foi realizado de acordo com Medronho \& Perez (2008), sendo considerado a RIFI como 100\% específica.

Para a análise estatística utilizou-se o programa Instat 3.0, onde os dados foram submetidos à tabela de contingência utilizando o Teste de Qui-quadrado, o nível de significância adotado foi de $5 \%$.

\section{Resultados}

Dos 59 animais sororreagentes avaliados 34 (62\%) eram oligossintomáticos, 19 (20\%) sintomáticos e 6 (16\%) assintomáticos. No teste parasitológico foram identificados 33 animais positivos (55,93\%) (Figura 1 e 2) e destes 20 pertenciam ao grupo oligossintomático (grupo 2) (60,60\%), 11 eram sintomáticos (grupo 3) (33,33\%) e 2 assintomáticos (grupo 1) (6,06\%) (Tabela1). Observou-se que os animais sintomáticos nem sempre eram positivos no teste parasitológico, assim como os assintomáticos nem sempre eram negativos. Não houve diferença estatística na positividade do teste de acordo com a sintomatologia clínica ( $\mathrm{p}=0,4995)$.

A sensibilidadeno diagnóstico parasitológico foi de $59 \%, 58 \%, 33 \%$ para os cães oligossintomáticos, sintomáticos e assintomáticos respectivamente.

No total obteve-se 177 imprints de amostras de baço, medula óssea e linfonodo, sendo que destes 73 foram positivos (41,24\%), com 28 amostras de medula óssea (38,35\%), 25 de baço (34,24\%) e 20 de linfonodo (27,39\%) (Tabela 2), não havendo diferença estatística entre os locais de coleta avaliados ( $\mathrm{p}=0,3191)$. Destarte a análise estatística, a medula óssea mostrou ser o local com maior frequência de positividade na avaliação parasitológica, podendo este órgão ser considerado ideal para a realização do exame.

A sensibilidade do teste de acordo com o local de coleta variou de $33,89 \%$ a $47,45 \%$, sendo 47,45\% na medula óssea 42,37\% no baço (42,37\%) 33,89\% no linfonodo.

De acordo com os dados demonstrados na Tabela 3, quando a leitura foi realizada por apenas um observador obteve-se 13 (22,03\%) animais positivos, já com dois observadores 33 (55,93\%), aumento extremamente significativo ( $\mathrm{p}=0,0003)$.

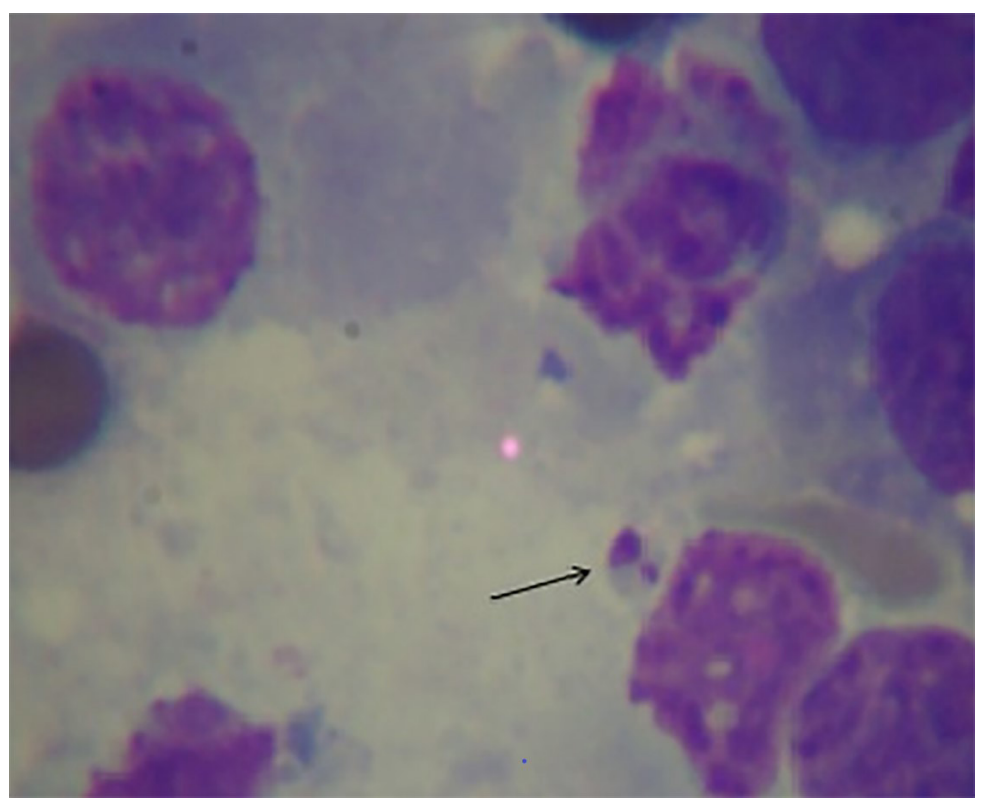

Figura 1. Parasitológico esfregaço de baço de cão soropositivo para leishmaniose. Forma amastigota livre (ponta da seta) com cinetoplasto, núcleo e citoplasma evidente em esfregaço de baço. Panótico Rápico, aumento de100x. 


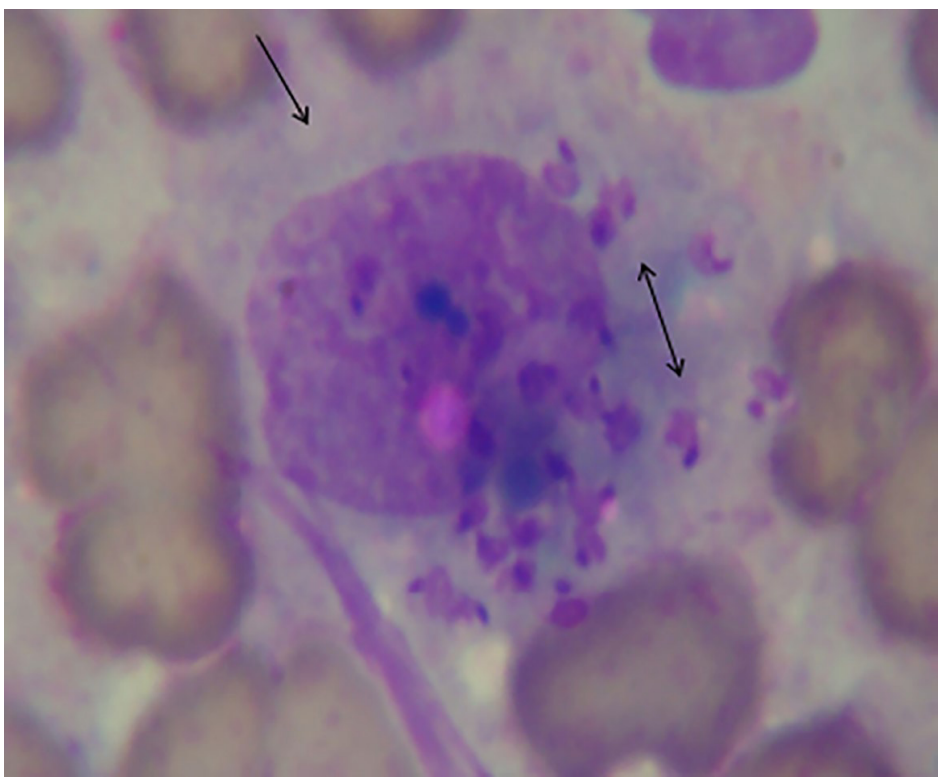

Figura 2. Parasitológico imprint de baço de cão soropositivo para leishmaniose. Macrófago (seta maior), contendo formas amastigotas (seta de duas pontas). Panótico Rápico, aumento de 100x.

Tabela 1. Número de animais positivos ou negativos no teste parasitológico de acordo com a sintomatologia clínica, Uberlândia - MG, 2010.

\begin{tabular}{ccccc}
\hline Classificação & Oligossintomático & Sintomático & Assintomático & Total \\
\hline Negativos & 14 & 8 & 4 & 26 \\
Positivos & 20 & 11 & 2 & 33 \\
Total & 34 & 19 & 6 & 59 \\
\hline
\end{tabular}

Tabela 2. Número de animais positivos e negativos de acordo com local de coleta de amostra para teste parasitológico, Uberlândia - MG, 2010.

\begin{tabular}{cccc}
\hline & Positivos & Negativos & Total \\
\hline Medula Óssea & 28 & 31 & 59 \\
Baço & 25 & 34 & 59 \\
Linfonodo & 20 & 39 & 59 \\
Total & 73 & 104 & 177 \\
\hline
\end{tabular}

Tabela 3. Sensibilidade do teste parasitológico em relação ao número de observadores, Uberlândia, 2010.

\begin{tabular}{cccc}
\hline Observadores & Positivos & Negativos & Total \\
\hline 2 & 33 & 26 & 59 \\
1 & 13 & 46 & 59 \\
\hline
\end{tabular}

\section{Discussão}

Sensibilidadee a especificidade dos testes para diagnóstico da LVC comoéum dos problemas mais desafiadores em relação a essa zoonose e os dados apresentados evidenciam essa problemática.

A sensibilidade do teste parasitológico de acordo com a sintomatologia foi avaliada por outros autores. Saridomichelakis et al. (2005), observaram sensibilidade de 94,7\% em cães sintomáticos e inferior a 30\% em oligossintomáticos. Moreira et al. (2007), encontraram sensibilidade de 75,6\% em 
cães sintomáticos, 32\% em oligossintomáticos e 39,1\% em assintomáticos. Saridomichelakis et al. (2005), afirmam que cães assintomáticos apresentam uma baixa carga parasitária, o que dificulta o diagnóstico parasitológico.

No presente estudo a maior sensibilidade observada quando o local de coleta foi medula ósseae a menor foi no linfonodo. Em trabalho realizado por Troncarelli et al. (2009), utilizando 100 animais, onde 51\% das amostras foram positivas no baço. Tasca et al. (2009), também observaram 48,15\% de positividade em 27 baços analisados.

Entretanto Saridomichelakis et al. (2005), analisando 68 cães sintomáticos demonstraram que o linfonodo foi positivo em 83,3\% dos cães e a medula óssea foi positiva em de 72,5\% e no grupo assintomático $12,5 \%$ dos cães foram positivos na análise de linfonodo e 7,8\% foram positivos para medula óssea. Quando utilizada uma técnica mais sensível em aspirados de baço e linfonodo, como a cultura, Barrouin-Melo et al. (2004), trabalhando com 64 animais demonstraram que o baço é o local de eleição sendo 97,9\% positivos contra 25\% de linfonodos.

Andrade et al. (2006), avaliando 39 cães obteve 33 amostras positivas no teste parasitológico, sendo 27 positivas em baço e linfonodo e 22 positivas para medula óssea. Na avaliação estatística do local de coleta, este autor não observou diferença entre baço, linfonodo e medula óssea, assim como na presente investigação.

Em trabalho realizado com humanos ao comparar aspirado de medula óssea com PCR, o aspirado obteve 55\% de sensibilidade contra 85\% do PCR (Piarroux et al., 1994). Brasil (2014), cita que o baço é o local mais sensível para a identificação do parasita seguido de medula óssea e por último o linfonodo, assim como da Silva et al. (2005), que ao trabalhar com humanos obteve 89\% de casos positivos em aspirados de medula óssea e 95\% em baço, recomendando que a medula seja o local de eleição por apresentar menos riscos ao paciente.

Houve um aumento significativo da sensibilidade do teste parasitológico quando realizado por dois observadores elevando-se em 33,9\%. Em 13 amostras positivas houve concordância pelos dois observadores. Estes números expressivos podem ser justificados pelo fato da sensibilidade do diagnóstico relacionar-se com o número de campos lidos sendo o ideal de 200 campos (Brasil, 2014) e também a influência do tempo. Segundo da Silva et al. (2005), ao compararem o exame parasitológico em relação ao tempo, obteve 57 (65,5\%) positivos com 5 minutos de leitura e quando a mesma estendeu-se por mais de 60 minutos obteve-se 87 (89\%) positivos para medula óssea de 98 pacientes.

\section{Conclusão}

Portanto, conclui-se que não houve influencia da sintomatologia clínica apresentada pelos animais e o exame parasitológico, assim como o local de coleta a ser utilizado. Porém a medula óssea mostrou ser local de eleição para coleta. Por outro lado o número de observadores foi determinante no aumento da sensibilidade do teste.

\section{Referências}

Abreu, C. R. D., Parpinelli, A. C., Lima, R. R. D., Dias, L. G. G. G., Pereira, L. D. F., \& Dias, F. G. G. (2015). Description of six autochthonous cases of canine visceral leishmaniasis diagnosed in Pedregulho (São Paulo, Brazil). Revista Brasileira de Parasitologia Veterinária, 24(2), 216-219. http://dx.doi.org/10.1590/S1984-29612015025. PMid:26154962.

Andrade, H. M., Reis, A. B., Santos, S. L., Volpini, A. C., Marques, M. J., \& Romanha, A. J. (2006). Use of PCR-RFLP to identify Leishmania species in naturally infected dogs. Veterinary Parasitology, 140(3-4), 231-238. http:// dx.doi.org/10.1016/j.vetpar.2006.03.031. PMid:16682124.

Barrouin-Melo, S. M., Larangeira, D. F., Trigo, J., Aguiar, P. H. P., Santos, W. L. C., \& Pontes-de- Carvalho, L. (2004). Comparison between splenic and lymph node aspirations as sampling methods for the parasitological detection of Leishmania chagasi infection in dogs. Memórias do Instituto Oswaldo Cruz, 99(2), 195-197. http:// dx.doi.org/10.1590/s0074-02762004000200014.

Brasil, Ministério da Saúde, Secretaria de Vigilância em Saúde, Departamento de Vigilância Epidemiológica. (2014). Manual de vigilância e controle da Leishmaniose Visceral. Brasília.

Gontijo, C. M. F., \& Melo, M. N. (2004). Leishmaniose visceral no Brasil: quadro atual, desafios e perspectivas. Revista Brasileira de Epidemiologia, 7(3), 338-349. http://dx.doi.org/10.1590/S1415-790X2004000300011. 
Mancianti, F., Gramiccia, M., Gradoni, L., \& Pieri, S. (1988). Studies on canineleishmaniasis control. 1. Evolution of infection of different clinical forms of canine leishmaniasis following antimonial treatment. Transactions of the Royal Society of Tropical Medicine and Hygiene, 82(4), 566-567. http://dx.doi.org/10.1016/0035-9203(88)90510-X. PMid:3076714.

Medronho, R. A., \& Perez, M. A. (2008). Epidemiologia (1. ed., p. 260). São Paulo: Atheneu.

Moreira, M. A. B., Luvizotto, M. C. R., Garcia, J. F., Corbett, C. E. P., \& Laurenti, M. D. (2007). Comparison of parasitological, immunological and molecular methods for the diagnosis of leishmaniasis in dogs with different clinical signs. Veterinary Parasitology, 145(3-4), 245-252. http://dx.doi.org/10.1016/j.vetpar.2006.12.012. PMid:17257764.

Peixoto, H. M., Oliveira, M. R. F., \& Romero, G. A. S. (2015). Serological diagnosis of canine visceral leishmaniasis in Brazil: systematic review and meta-analysis. Tropical Medicine \& International Health, 20(3), 334-352. http:// dx.doi.org/10.1111/tmi.12429. PMid:25403359.

Piarroux, R., Gambarelli, F., Dumon, H., Fontes, M., Dunan, S., Mary, C., Toga, B., \& Quilici, M. (1994). Comparison of PCR with direct examination of bone marrow aspiration, myeloculture, and serology for diagnosis of visceral Leishmaniasis in immunocompromised patients. Journal of Clinical Microbiology, 32(3), 746-749. PMid:8195388.

Saridomichelakis, M. N., Mylonakis, M. E., Leontides, L. S., Koutinas, A. F., Billinis, C., \& Kontos, V. I. (2005). Evaluation of lymph node and bone marrow cytology in the diagnosis of canine leishmaniasis (Leishmania infantum) in symptomatic and asymptomatic dogs. The American Journal of Tropical Medicine and Hygiene, 73(1), 82-86. PMid:16014839.

Silva, F. S. (2007). Patologia e patogênese da leishmaniose visceral canina. Revista Trópica: Ciências Agrárias e Biológicas, 1, 20-31.

Silva, M. R. B., Stewart, J. M., \& Costa, C. H. N. (2005). Sensitivity of bone marrow aspirates in the diagnosis of visceral leishmaniasis. The American Journal of Tropical Medicine and Hygiene, 72(6), 811-814. PMid:15964968.

Silva, S. M., Ribeiro, V. M., Ribeiro, R. R., Tafuri, W. L., Melo, M. N., \& Michalick, M. S. M. (2009). First report of vertical transmission of Leishmania (Leishmania) infantum in a naturally infected bitch from Brazil. Veterinary Parasitology, 166(1-2), 159-162. http://dx.doi.org/10.1016/j.vetpar.2009.08.011. PMid:19733439.

Solano-Gallego, L., Miró, G., Koutinas, A., Cardoso, L., Pennisi, M. G., Ferrer, L., Bourdeau, P., Oliva, G., \& Baneth, G. (2011). LeishVet guidelines for the practical management of canine leishmaniosis. Parasites \& Vectors, 4(1), 86. http://dx.doi.org/10.1186/1756-3305-4-86. PMid:21599936.

Tasca, K. I., Buzetti, W. A., Tenorio, M. S., Paulan, S. C., Lima, F. L., Queiroz, N. M., Machado, R. Z., Oliveira, T. M., Neves, M. F., Noronha Junior, A. C., \& Assis, J. (2009). Exames parasitológicos, imunoistoquímicos e histopatológicos para detecção de Leishmania chagasi em tecidos esplênicos de cães com Leishmaniose Visceral. Revista Brasileira de Parasitologia Veterinária, 18(1), 27-33. http://dx.doi.org/10.4322/rbpv.01801005. PMid:19500457.

Troncarelli, M. Z., Lucheis, S. B., Camargo, J. B., Machado, J. G., \& Langoni, H. (2009). Análise clínica elaboratorial em cães eutanasiados no centro de controle de zoonoses de Bauru-SP, com vistas ao diagnóstico da leishmaniose visceral (LV). Veterinária e Zootecnia, 16, 343-353.

World Health Organization. (2010). Working to overcome the global impact of neglected tropical diseases: first WHO report on neglected tropical diseases. Genebra. Recuperado em 1 de fevereiro de 2016, de http://apps. who.int/iris/handle/10665/44440 